Abstract P051 Table 1 Parent-reported symptoms of OSAHS

\begin{tabular}{|l|l|l|l|}
\hline \multicolumn{1}{|c|}{ Symptoms } & \multicolumn{1}{|c|}{ Present } & \multicolumn{1}{|c|}{ Not present } & \multicolumn{1}{|c|}{ Not known } \\
\hline Snoring & $30(75 \%)$ & $6(15 \%)$ & $4(10 \%)$ \\
\hline Apneas & $16(40 \%)$ & $19(47.5 \%)$ & $5(12.5 \%)$ \\
\hline Arousals & $21(52.5 \%)$ & $14(35 \%)$ & $5(12.5 \%)$ \\
\hline Mouth breathing & $26(65 \%)$ & $8(20 \%)$ & $6(15 \%)$ \\
\hline Morning headache & $14(35 \%)$ & $21(52.5 \%)$ & $5(12.5 \%)$ \\
\hline Daytime sleepiness & $22(55 \%)$ & $14(35 \%)$ & $4(10 \%)$ \\
\hline Concentrating difficulty & $19(47.5 \%)$ & $17(42.5 \%)$ & $4(10 \%)$ \\
\hline Behavioral problems & $9(22.5 \%)$ & $26(65 \%)$ & $5(12.5 \%)$ \\
\hline
\end{tabular}

Abstract P051 Table 2 Cardiorespiratory polygraphy parameters

\begin{tabular}{|l|l|l|l|}
\hline \multicolumn{1}{|c|}{ Parameter } & \multicolumn{1}{|c|}{ Mean } & \multicolumn{1}{c|}{ SD } & \multicolumn{1}{c|}{ Range } \\
\hline Oxygen saturations (\%) & 96.6 & \pm 1.31 & $93-98.6$ \\
\hline SpO2 (\%) & 90.18 & \pm 3.89 & $79-95$ \\
\hline TcPCO2 (kPa) & 5.89 & \pm 0.325 & $5.2-6.56$ \\
\hline ODI/hr & 3.38 & \pm 4.43 & $0-17$ \\
\hline oAHI/hr & 0.745 & \pm SD 1.55 & $0-6.5$ \\
\hline
\end{tabular}

were collected. Data analysis was undertaken using Prism 8.0 (GraphPad, USA).

Results 40 patients (75\% male) were reviewed. Mean age at time of study was eight years (SD \pm 4.24). Mean BMI $21.24 \mathrm{~kg} / \mathrm{m}-2$ (SD \pm 6.99). Treatment burden was variable; $39 / 40$ patients were on inhaled corticosteroids with 4 patients on biologicals. At the time of the study, concerns about treatment adherence were present in at least $25 \%$ of patients.

Out of eight parent-reported symptoms (table1), snoring was the commonest symptom and mean Cardiorespiratory Polygraphy Parameters (table 2) were within the reference range except for ODI. 2(5\%) patients had oAHI $>5$.

oAHI positively correlated with parent-reported symptom score $(r=0.33, p=0.03)$ and ODI $(r=0.400, p=0.01)$. Neither mean TcPCO2 $(\mathrm{p}=0.624)$ nor modified Epworth score $(p=0.352)$ correlated significantly with oAHI.

Discussion A focused symptom analysis with oximetry to calculate ODI is useful for predicting OSAHS in children with asthma. This could conveniently be performed at home instead of level 2 studies being a first-line investigation.

\section{P052 ELECTRICAL STIMULATION AS A THERAPEUTIC APPROACH IN OBSTRUCTIVE SLEEP APNOEA - A SYSTEMATIC REVIEW AND META-ANALYSIS}

${ }^{1}$ Culadeeban Ratneswaran* ${ }^{1,2}$ Ahmad Guni, 1,2,3 Martino F Pengo, ${ }^{1,4}$ Miral Al-Sherif,,${ }^{1,5}$ Baiting He, ${ }^{1,6}$ Michael CF Cheng, ${ }^{1,2}$ Joerg Steier, ${ }^{1,7}$ Esther I Schwarz. 'Guy's and St Thomas' NHS Foundation Trust, London, UK; ${ }^{2}$ King's College London, London, UK; ${ }^{3}$ University of Milan, Milan, Italy; ${ }^{4}$ University of Minia, Minia, Egypt; ${ }^{5}$ Guangzhou Medical University, Guangzhou, China; ${ }^{6}$ University of Sydney, Sydney, Australia; 'University of Zurich, Zurich, Switzerland

\subsection{6/bmjresp-2019-bssconf.52}

Introduction Electrical stimulation of the upper airway dilator muscles is an emerging treatment for obstructive sleep apnea (OSA). Hypoglossal nerve stimulation (HNS) has recently been approved as treatment alternative to continuous positive airway pressure (CPAP) for selected patients with moderate to severe OSA, while transcutaneous electrical stimulation (TES) of the upper airway is being investigated as non-invasive alternative.

Methods A systematic review and meta-analysis (CRD42017074674) including literature published up to May 2018 has been performed to investigate the impact of HNS 
and TES on OSA severity assessed by the apnoea-hypopnoea index (AHI) and the Epworth Sleepiness Scale (ESS) as measure of subjective sleepiness. Random-effects models were used. Heterogeneity and between study variance were assessed by $\mathrm{I} 2$ and $\tau 2$, respectively. The Robins- 1 tool was utilised to assess the risk of bias for each study.

Results Of the 41 clinical trials identified $(n=1,853)$, data from 20 trials $(n=895)$ could be pooled for meta-analysis (15 HNS [n=808], 5 TES $[n=87])$. Patient were predominantly middle aged (mean \pm SD $56.9 \pm 5.5$ years), overweight (body mass index, BMI $29.1 \pm 1.5 \mathrm{~kg} / \mathrm{m} 2$ ) with severe OSA (AHI 37.5 $\pm 7.0 \mathrm{~h}-1$ ) and were followed-up for $6.9 \pm 4.0$ month (HNS) and $0.2 \pm 0.4$ months (TES), respectively. The AHI improved by -24.9 [95\%CI $-28.5,-21.2] \mathrm{h}-1$ in HNS ( $\chi 279 \%$, I $282 \%)$ and by -16.5 [95\%CI $-25.1,-7.8] \mathrm{h}-1$ in TES $(\chi 27 \%$, I 2 43\%; both $\mathrm{p}<0.001)$. The ESS was reduced by $-5.0(95 \%$ CI $-5.9,-4.1)$ points $(p<0.001)$. Nineteen of trials were non-randomised studies and were found to be at a moderate or serious risk of bias favouring treatment.

Discussion Both invasive and transcutaneous electrical stimulation reduce OSA severity by a clinically relevant margin. HNS results in a clinically relevant improvement of symptoms. However, data are mainly based on non-randomised trials.

\section{P053 MANUAL SCORING VS THE INBUILT AUTOMATED SCORING SYSTEM OF OVERNIGHT MULTI-CHANNEL SLEEP STUDIES (NOX T3); EVALUATING THE NEED TO MANUALLY SCORE FOR OSAHS}

Liam O'Reilly*, Gemma Cramp, Michelle Goodlad. UHCW NHS Trust, Coventry, UK

\subsection{6/bmiresp-2019-bssconf.53}

Introduction Current practice at University Hospital Coventry (UHCW) is for qualified, peer reviewed physiologists to manually score multi-channel sleep studies. Apnoea-Hypopnea Index (AHI) or Oxygen Desaturation Index (ODI) used to diagnose sleep disordered breathing and categorise severity. Analysis programs incorporated into device software allows parameters to be scored automatically (AS) without the need for manual scoring (MS) reducing waiting times of analysis reducing time from referral to treatment.

Aim To determine the accuracy and reliability of the AS systems compared with MS and establish if this can be implemented to diagnose sleep disordered breathing.
Methods 121 multi-channel sleep studies (NOX T3) from the department's Nocturnal diagnostic database (Version 5.1.3, Nox Medical, Reykjavik, Iceland). Each study initially MS were re-analysed using AS. The MS AHI governed the subgroup $($ Normal $=\mathrm{AHI}<5$, Mild $=$ AHI 5-14, Moderate $=$ AHI 15-29, Severe $=$ AHI $>30)^{1}$ Agreement measurements used Wilcoxon signed rank test and Spearman's rank-order correlation.

Results Figure 1 manual scoring produced higher values across all sub-groups for AHI. Spearman's coefficient showed a strong correlation in all parameters for severe sub-group, scoring of hypopneas showed weakest correlation. Significant differences were seen for moderate and severe AHI, no significant differences seen in normal and mild categories (table 1). 22 studies changed severity when AS performed, 12 changing clinical outcome.

Conclusion AS is reliable scoring severe AHI compared to MS but underscored studies showing false negatives. No clinical differences in the outcomes despite statistical differences seen. AS AHI of $15 /$ hour showed no change to clinical outcomes suggesting MS is not required. Current study shows MS required when AS shows AHI $<15 / \mathrm{hr}$ as hypopneas are underscored producing false negatives. Larger sample sizes are needed to change clinical practice. Further research using different devices needed.

\section{REFERENCES}

1. Sleep-related breathing disorders in adults: recommendations for syndrome definition and measurement techniques in clinical research. The Report of an American Academy of Sleep Medicine Task Force. Sleep Aug 1 1999;22(5):667-689.

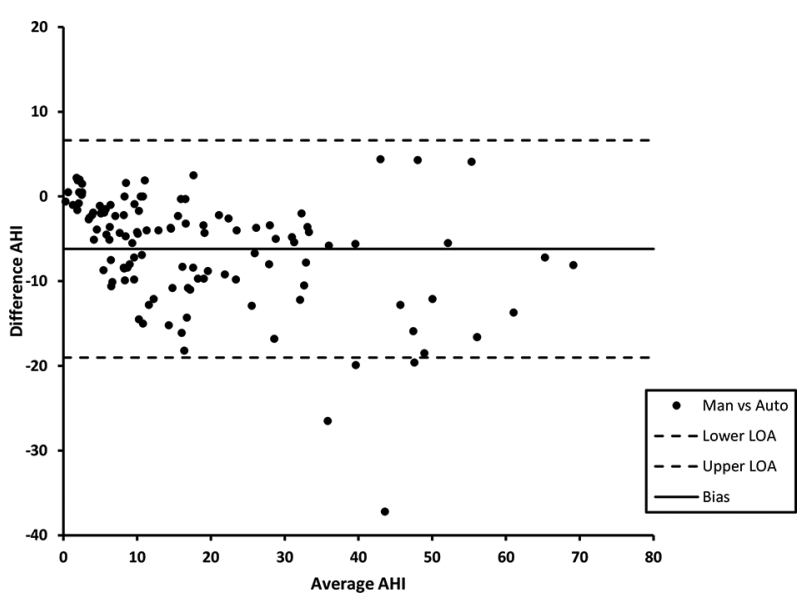

Abstract P053 Figure 1 Bland Altman plot showing the agreement of manual vs automated scoring of NOX T3

Abstract P053 Table 1 The median difference (IQR) between the manual and automated scoring system showing correlation and significance

\begin{tabular}{|c|c|c|c|c|c|c|c|c|c|c|c|c|}
\hline & \multicolumn{4}{|c|}{ Median Difference (IQR) } & \multicolumn{4}{|c|}{ r Manual v Auto Scoring } & \multicolumn{4}{|c|}{ p Manual v Auto Scoring } \\
\hline & $\mathrm{AHI}$ & Apnoea & Hypopnea & ODI & $\mathrm{AHI}$ & Apnoea & Hypopnea & ODI & $\mathrm{AHI}$ & Apnoea & Hypopnea & ODI \\
\hline NORMAL & $-0.20(2.43)$ & $1.00(1.53)$ & $-1.00(1.60)$ & $-1.10(1.83)$ & -0.1 & 0.2 & 0.6 & 0.7 & 0.500 & 0.001 & $<0.001$ & $<0.006$ \\
\hline MILD & $-1.50(7.4)$ & $-0.20(1.50)$ & $0.10(9.20)$ & $-0.40(8.30)$ & -0.2 & 0.7 & -0.2 & 0.1 & 0.153 & 0.022 & 0.285 & 0.979 \\
\hline MODERATE & $-8.40(7.6)$ & $0.20(3.30)$ & $-5.50(9.20)$ & $-8.60(5.50)$ & 0.4 & 0.9 & 0.4 & 0.6 & $<0.001$ & 0.604 & $<0.001$ & $<0.001$ \\
\hline SEVERE & $-8.10(11.05)$ & $-0.40(7.70)$ & $-6.20(8.80)$ & $-8.90(9.75)$ & 0.7 & 0.9 & 0.9 & 0.7 & $<0.001$ & 0.863 & 0.866 & 0.737 \\
\hline
\end{tabular}

